\title{
DISEÑO Y CONSTRUCCIÓN DE UN ESCÁNER BIFOCAL PARA LA OBTENCIÓN DE UNA NUBE DE PUNTOS A TRAVÉS DE FILTRADO Y ADQUISICIÓN DE IMÁGENES
}

\author{
Jorge Yánez Cajas ${ }^{1}$, Juan Musuña Toapanta ${ }^{2}$, Luis Oñate Cadena ${ }^{3}$ y \\ Bayardo Campusano Nieto ${ }^{4}$
}

\section{Resumen}

En este artículo se presenta el diseño y la implementación de un escáner bifocal para realizar la reconstrucción de imágenes 3D a partir de imágenes en 2D. El escáner está constituido por una base giratoria bifocal compuesta de 2 cámaras web y un láser, la misma que capturará múltiples imágenes desde diferentes ángulos, para luego ser procesadas y digitalizadas. En una etapa posterior se aplicarán técnicas de visión artificial a fin de eliminar ruido, ajustar el brillo, resaltar bordes, mejorar la imagen en escala de grises y seleccionar la región de interés. El proceso de selección de la región de interés indicará los valores de la posición en las coordenadas en $X, Y$, $Z$, siendo esta la nube de puntos que se desea encontrar. Esto permite la reconstrucción de 3D a partir de una imagen $2 \mathrm{D}$ a través de visión estereoscópica, ya que su análisis se lo realiza por medio de rebanadas o secciones de las imágenes capturadas.

Palabras clave: Visión estereoscópica, procesamiento de imágenes, nube de puntos, regiones de interés.

\begin{abstract}
The project aims at building a bifocal swivel base made of 2 web cameras and a laser. It will capture multiple images from different angles and then be processed and digitized. At a later stage computer vision techniques were applied to remove noise, adjust brightness, highlight edges, improve the image in grayscale, and select the region of interest. The selection process of the region of interest values indicate the position coordinates of $X, Y, Z$, this being the point cloud to be found. This allows the reconstruction of 3D from a 2D image through stereoscopic vision, as their analysis is performed by means of slices or sections of the captured images.
\end{abstract}

Keywords: Stereoscopic vision, image processing, point cloud, regions of interest.

\footnotetext{
${ }^{1}$ Estudiante de Ingeniería Electrónica - Universidad Politécnica Salesiana.

${ }^{2}$ Estudiante de Ingeniería Electrónica - Universidad Politécnica Salesiana.

${ }^{3}$ Ingeniero en Electrónica y Telecomunicaciones, Docente de la Universidad Politécnica Salesiana, sede Quito

${ }^{4, *}$ Físico, Especialista en Visión Artificial - Universidad Politécnica Salesiana, Docente - UPS - sede Quito. Autor para correspondencia gcampuzano@ups.edu.ec
}

Recibido: 31-01-2014, Aprobado tras revisión: 08-04-2014.

Forma sugerida de citación: Yánez, J., Musuña, J., Onãte, L. y Campusano, G. (2014). "Diseño y construcción de un escáner bifocal para la obtención de una nube de puntos a través de filtrado y adquisición de imágenes ". INGENIUS. N. ${ }^{\circ} 11$, (Enero-Junio). pp. 18-24. ISSN: 1390-650X. 


\section{Introducción}

La utilización de robots en diversos campos se ha vuelto muy importante en la actualidad y más aún cuando se han incorporado sistemas de visión artificial que permiten digitalizar imágenes de diversas escenas y objetos en el computador [1]. Una de las aplicaciones más interesantes de la visión artificial es la reconstrucción tridimensional de objetos pequeños partiendo del tratamiento de imágenes [2]. En dicho proceso se busca generar una nube de puntos que posteriormente permita reconstruir la imagen en 3D. En tal virtud, en este trabajo se propone llevar a cabo dicha tarea por medio de dos cámaras web, un láser y un programa informático. Con ello, se podrá llevar a cabo la reconstrucción en 3D del objeto escaneado y a la vez, se podrán corregir los errores de oclusiones o puntos ciegos de los objetos presentados en [3]. Estas correcciones se podrán llevar a cabo ya que cada cámara tiene su posición y ángulo de captura, lo que posibilitará que cada una de ellas capte partes del objeto que no puede capturar la otra.

Asimismo, es importante mencionar que la reconstrucción de objetos pequeños es una tarea factible, y por el contrario, para reconocer objetos de mayor tamaño se requerirá desarrollar un mecanismo robótico más complejo.

\section{Escáner tridimensional bifocal}

Un escáner bifocal busca eliminar las oclusiones o puntos ciegos de las figuras cóncavas. Esto se logra con dos cámaras web y un láser colocados sobre una superficie plana. Las cámaras antes mencionadas tienen diferente ángulo de captura una respecto a la otra, aspecto que permite que se pueda capturar partes poco visibles del objeto.

El movimiento de la base giratoria del escáner puede ser manual (izquierda-derecha) o automático y se realiza sobre un mismo eje a través de un motor como se muestra en la Figura 1.
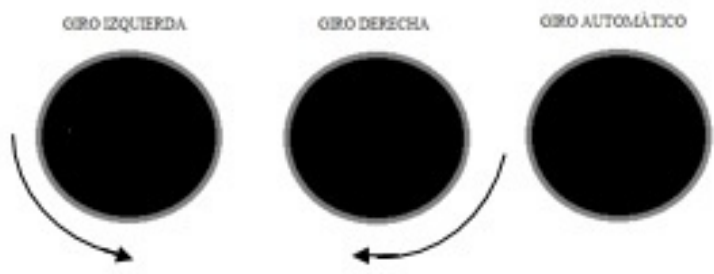

Figura 1. Movimiento del escáner bifocal.

\subsection{Sensores ópticos}

Son aquellos que detectan un objeto a través de un haz de luz. Esta detección se produce cuando este penetra una rueda segmentada, que llega al receptor y cambia el estado de salida convirtiendo la luz en pulsaciones en valores de 1 y 0 digital, permitiendo saber cuántas revoluciones da en 360 grados, como se ilustra en la Figura 2 [4].

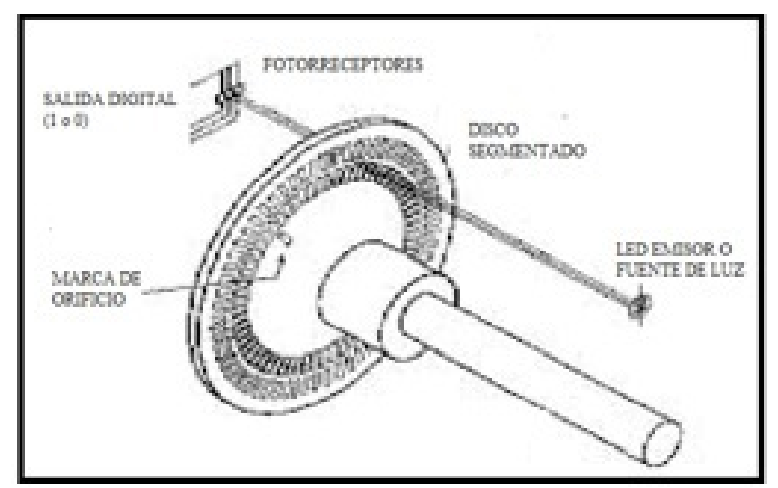

Figura 2. Componentes de codificador óptico para medir el ángulo de rotación del escáner bifocal [5].

\subsection{Rotación de la base giratoria}

Es importante hallar el ángulo de giro, ya que este establece una relación directa entre la constante de calibración y cada pixel en $X$ de la figura escaneada.

Mediante la ecuación 1 podemos obtener el ángulo de desplazamiento de la base giratoria sobre su propio eje de rotación, de acuerdo a los 360 grados que debe girar hasta completar su trayectoria circular. El valor del ángulo de desplazamiento dependerá del número de segmentos de la base giratoria (PPV) que va censar el encoder.

$$
\varnothing=\frac{360^{\circ} \times C}{P P V}
$$

Donde:

$\varnothing: \quad$ ángulo de rotación de la base giratoria.

$P P V$ : segmentos de la base giratoria.

$C: \quad$ pulsaciones por recorrido.

\subsection{Calibración de cámaras}

En la etapa de calibración es donde se normaliza la posición de las cámaras con respecto a su base giratoria, a fin de determinar la distancia del láser hasta el borde izquierdo o derecho del cilindro de calibración $\left(D_{c}\right)$ y la coordenada en $X$ del láser con respecto a las cámaras $\left(X_{c}\right)$ [3]. Estas constantes se emplearán posteriormente para encontrar la coordenada de profundidad del objeto $\left(Z_{m}\right)$ y por ello se usaron dos 
métodos de calibración: a) luz láser y pantalla y b) cuerpo de calibración.

\subsubsection{Luz láser y pantalla}

Esta calibración se la realiza colocando verticalmente una hoja blanca de cualquier color sobre la base giratoria, en la misma estará proyectada la luz láser. La visión de las dos cámaras web tiene que centrarse en la proyección del láser, el mismo que permitirá determinar la coordenada en $X$ del centro del láser [5]. En las Figuras 3 y 4 se muestran los puntos de calibración anteriormente mencionados de las dos cámaras y el láser.

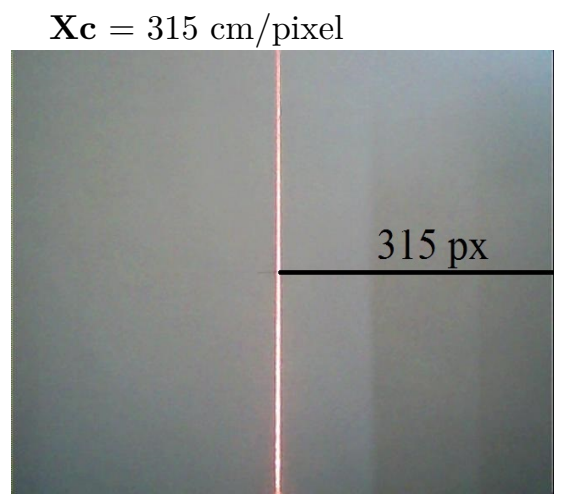

Figura 3. Centro de calibración de la cámara derecha.

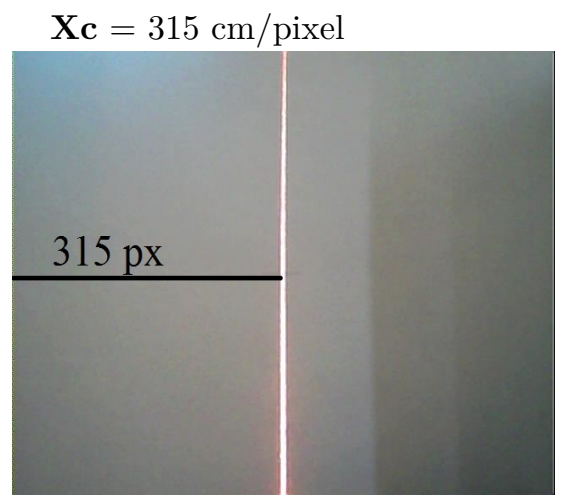

Figura 4. Centro de calibración de la cámara izquierda.

\subsubsection{Cuerpo de calibración}

Este método consiste en proyectar el láser sobre un cilindro de madera o metal, esto se realiza porque el cilindro es simétrico y mantendrá la misma forma durante el giro, lo que permite determinar la relación entre el sistema de referencia del objeto y el de la cámara. En la Figura 5 se aprecia el proceso realizado.

Una vez establecido el sistema procedemos al análisis y cálculo de coordenadas necesarias para la reconstrucción tridimensional. Para ello utilizaremos las ecuaciones 2 y 3 y procedemos a calcular $D_{c}$ y $D_{m}$.
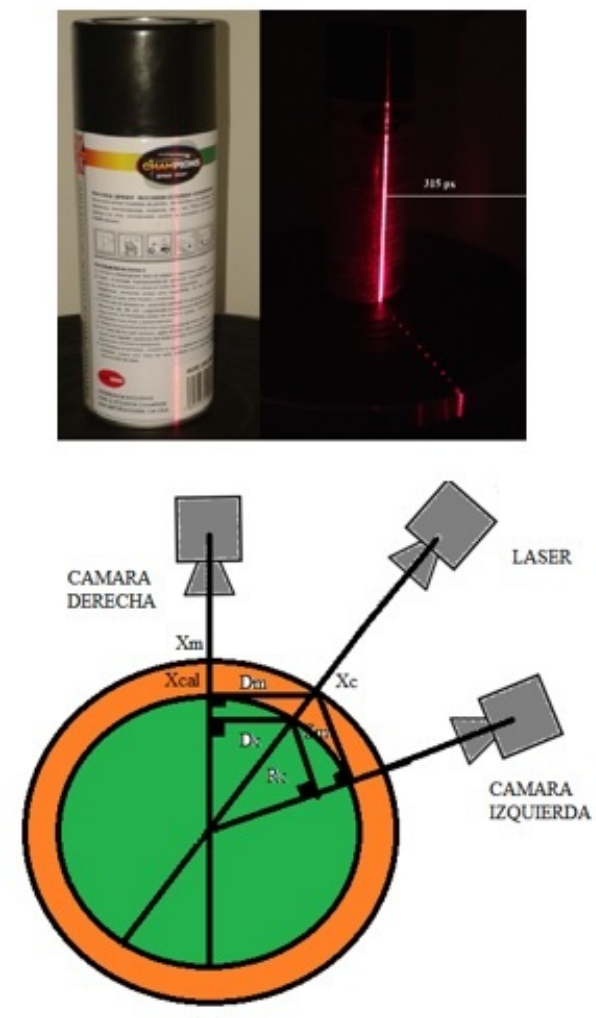

Figura 5. Componentes que se toman en cuenta en la calibración con cuerpos un cilindro.

$$
\begin{aligned}
& D_{c}=\left|X_{c a l}-X_{c}\right| \\
& D_{c}=\left|X_{m}-X_{c}\right|
\end{aligned}
$$

Donde:

$X_{c a l}$ : coordenada en $X$ del borde del cuerpo de calibración.

$X_{c}$ : coordenada en $X$ del láser con respecto a las cámaras

$X_{m}$ : coordenadas del borde del objeto escaneado.

A fin de determinar la coordenada $Z_{m}$ del objeto escaneado se aplica semejanza de triángulos (Figura 6) y se procede como se detalla en la ecuación 4:

$$
\begin{gathered}
\frac{D_{m}}{Z_{m}}=\frac{D_{c}}{R_{c}} \\
Z_{m}=\frac{D_{m} \times R_{c}}{D_{c}}
\end{gathered}
$$

Donde:

$Z_{m}$ : coordenada de profundidad del objeto. 


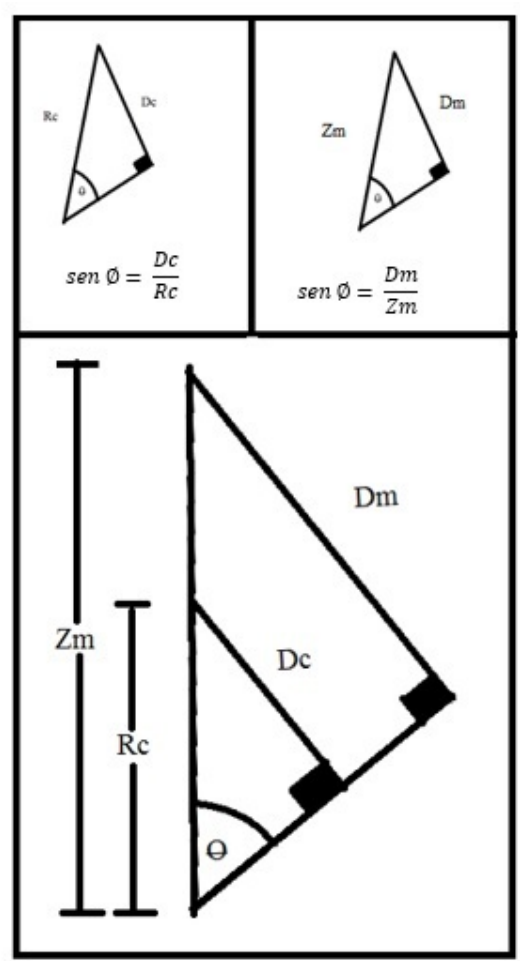

Figura 6. Semejanza de triángulos rectángulos.

\section{Desarrollo del sistema}

\subsection{Diseño del hardware}

En la Figura 7 se presenta el diagrama de flujo del hardware utilizado para el movimiento de la base giratoria, así como la adquisición de imágenes y procesamiento de las mismas. El control del giro (izquierda-derecha o automático) se realiza empleando un microcontrolador Atmega, mientras que las capturas de las imágenes de cada una de las cámaras (izquierda-derecha) se llevan a cabo a través de un programa desarrollado en Labview. Las imágenes se almacenarán para posteriormente filtrarlas, procesarlas y conseguir la reconstrucción tridimensional.

La comunicación entre la computadora y el microcontrolador se realiza de forma serial, empleando una velocidad de 9600 baudios. A fin de gestionar el giro automático o manual, el microcontrolador recibe información desde la computadora y con ella activa o desactiva los relés que forman parte de un puente $H$, obteniendo el giro deseado.

Asimismo, es importante indicar que el motor de la base giratoria tiene un encoder que permite conocer el ángulo de giro de la rueda segmentada a través de un haz de luz. Dicho haz llega al receptor y se convierte en pulsaciones entre 1 y 0 digital, contabilizando el número de pulsaciones realizadas en los 360 grados de giro. Las cámaras web son conectadas a los puertos

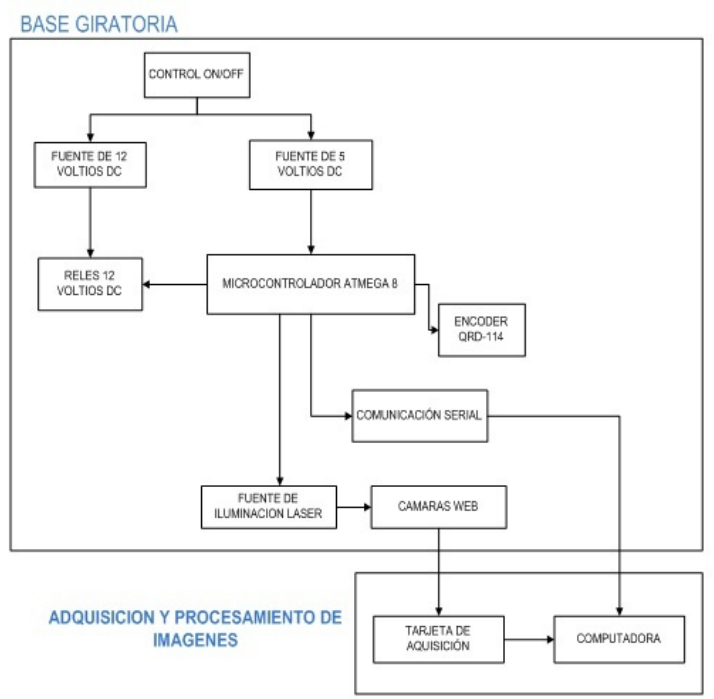

Figura 7. Diagrama de bloques del sistema de escáner tridimensional.

USB del computador y enlazadas a las librerías de Labview, a fin de visualizar el objeto a escanear y así poder capturar las imágenes que van a ser almacenadas en el computador, para su posterior tratamiento. La fuente de iluminación tiene un control de encendido y apagado independiente, que permitirá que esté encendido permanentemente en caso de ser necesario.

\subsection{Diseño del software}

En esta sección se presentan los diagramas de flujo de la etapa de adquisición de imágenes, así como del procesamiento de las mismas.

\subsubsection{Filtrado de imágenes}

En la Figura 8 se presenta el método que se utilizó para el procesamiento de imágenes, a fin de poder extraer el haz de luz de la figura.

En esta etapa se exportan, una a una, las imágenes anteriormente capturadas, a fin de aplicar los filtros a cada una ellas, aspecto que permite corregir contraste, brillo y eliminar secciones no deseadas de la escena [6]. Aplicando los filtros anteriores podemos separar el haz de luz de la fotografía, el mismo que nos permitirá obtener los puntos o coordenadas de la región de interés para luego exportarlos en un archivo de localización visual (VLS) y proseguir con su reconstrucción tridimensional.

\subsubsection{Determinación de la nube de puntos del objeto escaneado}

En esta etapa se leerá cada uno de los archivos generados en el proceso anterior a fin de reconstruir el objeto tridimensional. Para llevar a cabo esta tarea, se ingresan las siguientes variables: datos de calibración 
de la ecuación (2), el radio del cilindro de calibración $R_{c}$ y el número de fotografías a analizarse. A continuación se generan los puntos " $x$ " e " $y$ " de la imagen y a través de la ecuación 4 se obtiene la coordenada " $z$ ", se genera la nube de puntos, y vuelve a esperar un número de imágenes a procesar. En la Figura 9, se presenta en detalle el algoritmo utilizado para generar la nube de puntos.

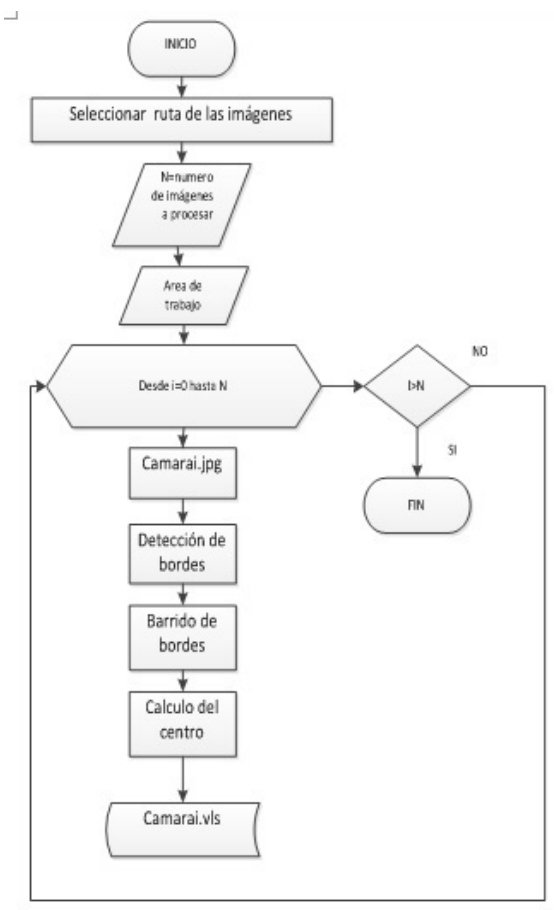

Figura 8. Parámetros de comunicación serial-computador.

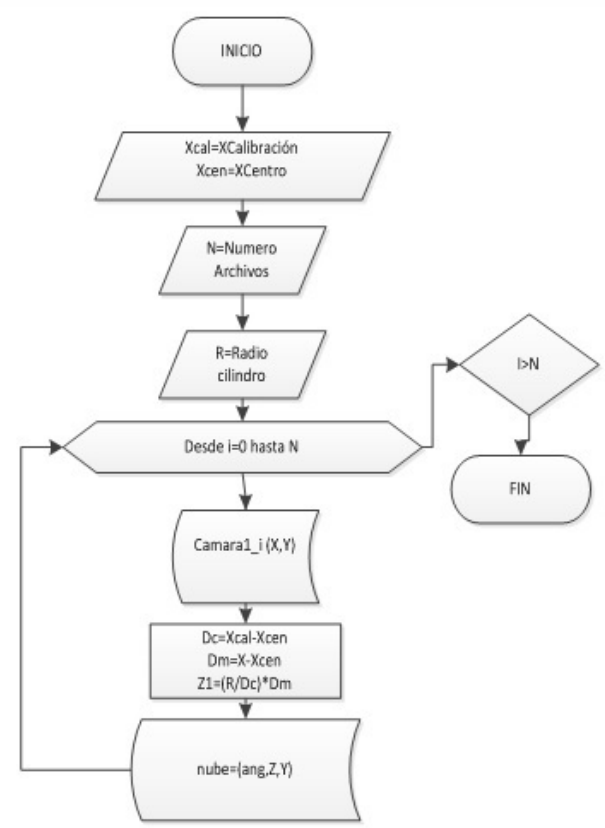

Figura 9. Diagrama de flujo para determinar la nube del objeto.

\subsubsection{Dispositivos y equipos empleados para la fabricación del escáner}

En la Figura 10 se puede observar la placa de control, mientras que en la Figura 11 se presenta el escáner bifocal tridimensional armado en su totalidad.

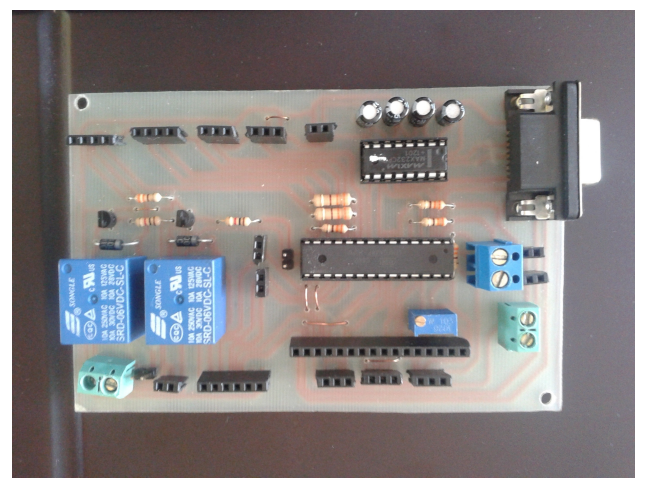

Figura 10. Placa de control.

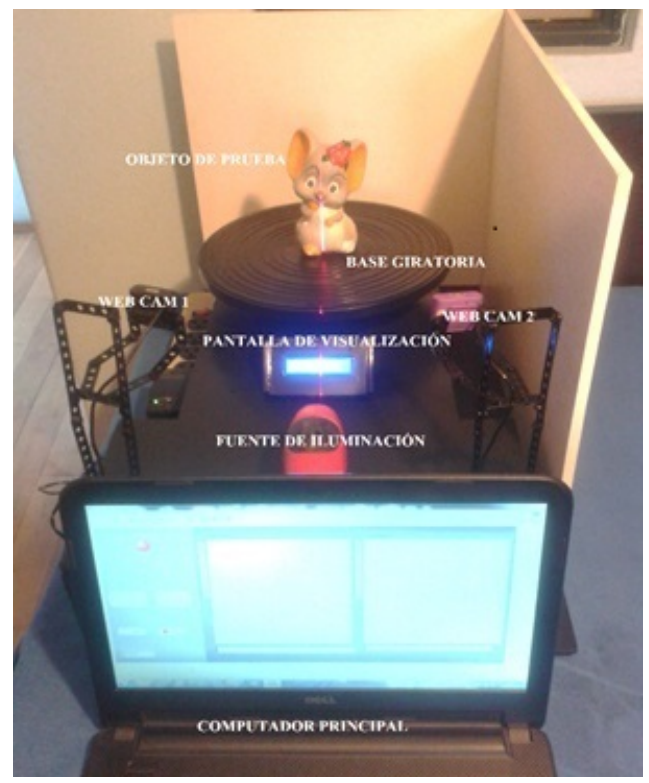

Figura 11. Escáner bifocal tridimensional.

\section{Análisis y resultados}

\subsection{Error del ángulo de giro de la base}

Este error se presenta en el ángulo de giro de la base, ya que las pulsaciones emitidas por el motor en su recorrido sobrepasan los 360 grados, por lo tanto:

$n C=$ número de pulsaciones en su recorrido

$$
\begin{gathered}
360^{\circ}-50 n C \\
366.5^{\circ}-x=50.9 n C \\
50 n C-100 \%
\end{gathered}
$$




$$
\begin{gathered}
50 n C-x=101.8 \% \\
101.8 \%-100 \%=1.8 \%
\end{gathered}
$$

Porcentaje de error en el $1.8 \%$ ángulo de giro

\subsection{Resultados}

A fin de verificar el correcto funcionamiento del sistema propuesto se realizaron pruebas de escaneo de diversas figuras. En este apartado se presentan dos en concreto, una primera con un jarrón que posee dimensiones de $15 \times 10 \mathrm{~cm}$ y la segunda con oso de peluche de similar tamaño. En la Figura 12 se puede apreciar del objeto escaneado por la cámara derecha.
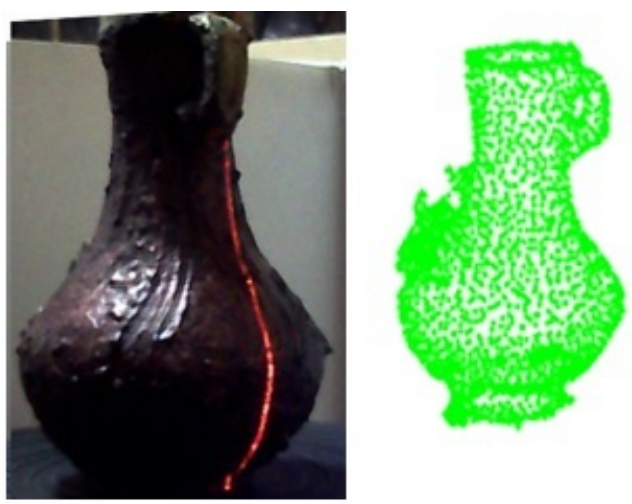

Figura 12. Vista de la vasija obtenida con la cámara derecha.

Asimismo, el objeto escaneado por la cámara izquierda se observa en la Figura 13.
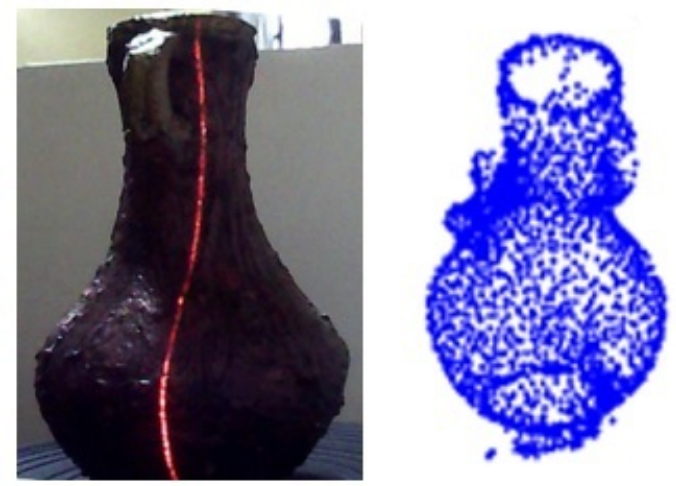

Figura 13. Vista de la vasija obtenida con la cámara izquierda.

En la Figura 14 se presenta el resultado de la unión de la nube de puntos de las cámaras izquierda y derecha. Se observa que este proceso permite completar objeto y eliminar las oclusiones que presentan las cámaras.

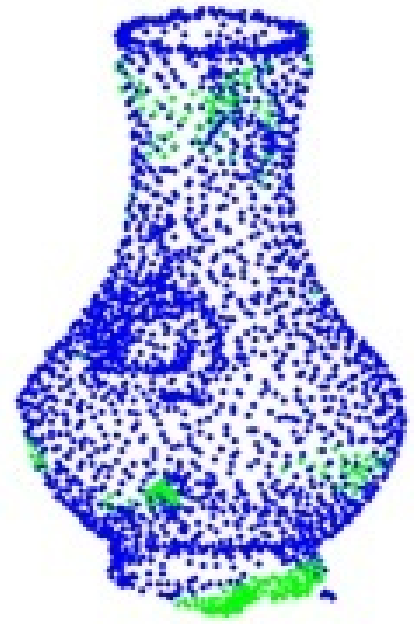

Figura 14. Vista tridimensional de la vasija.

De igual forma, también se realizó el proceso de escaneo para el oso de peluche. En las Figuras 15 y 16 se presentan los resultados obtenidos por las cámaras derecha e izquierda, respectivamente.

Finalmente, en la Figura 17 se presenta el resultado de la unión de la nube de puntos completo.
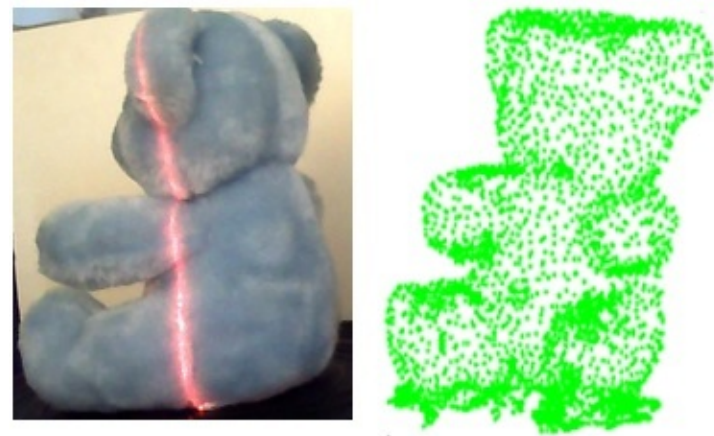

Figura 15. Vista del oso obtenida con la cámara derecha.

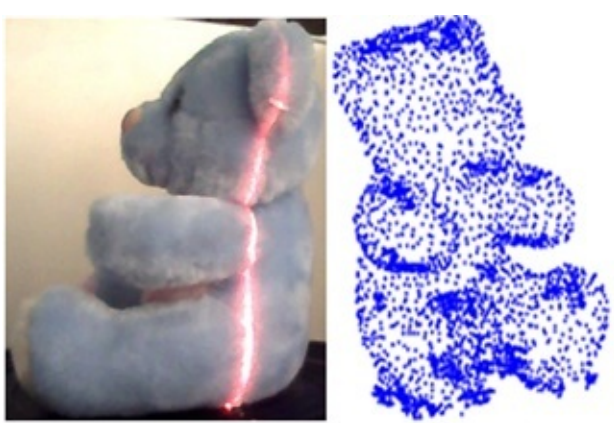

Figura 16. Vista del oso obtenida con la cámara izquierda. 


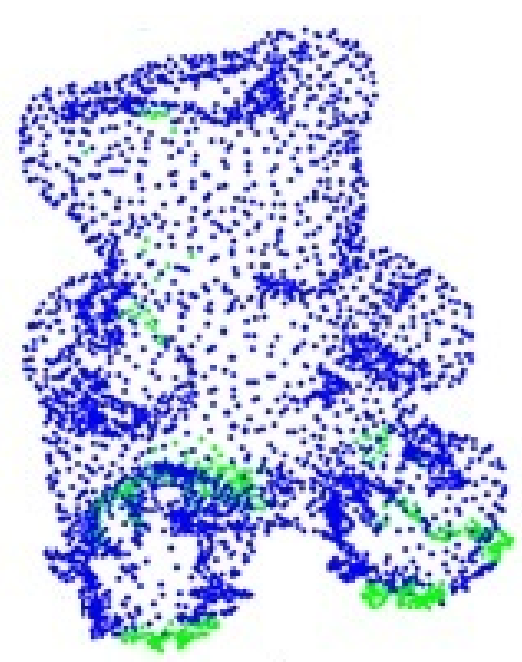

Figura 17. Vista tridimensional del oso.

\section{Conclusiones}

Es importante indicar que el equipo tendrá que estar correctamente calibrado en sus coordenadas iniciales, ya que de estas depende la toma de datos. Asimismo, para adquirir los datos se parte desde el mismo sistema de coordenadas, ya que si se cambia la posición del láser o cámaras, las imágenes tienden a salir en diferentes ángulos de rotación.

Para el escaneo de figuras se recomienda que tengan una altura máxima de $25 \mathrm{~cm}$ y mínimo de $10 \mathrm{~cm}$, a fin de evitar la obtención de falsos datos que pueden afectar la reconstrucción tridimensional del objeto.

También se pudo apreciar que el ángulo de giro influye mucho en la toma de datos, ya que al momento de obtener imágenes de una cámara con respecto a la otra, existirá un desfase de rotación de la nube de puntos, en consecuencia si el error es mayor se presentará solapamiento o distorsión de los puntos.

Mediante el escaneo de varios objetos se pudo observar que estos presentan secciones curvas o cóncavas. En estos casos una de las cámaras no podía captar dichas secciones, sin embargo, la otra cámara permitía solucionar este inconveniente.

\section{Referencias}

[1] G. Pajares and J. de la Cruz García, Visión por computador. Imágenes Digitales y Aplicaciones. $2 a$ Edición. RA-MA S.A. Editorial y Publicaciones, 2007.

[2] M. Domingo, Visión por computadora, U. C. de Chile, Ed. Departamento de Ciencia de la Computación, 2004.

[3] C. García and E. Narváez, "Sistema prototipo de scanner óptico tridimensional," Proyecto previo a la obtención del título de Ingeniero Electrónico, Universidad Politécnica Salesiana Sede Quito-Sur, Ecuador, 2007.

[4] National Instruments. Visión general de un codificador y sus aplicaciones. [Online]. Available: http://www.ni.com/white-paper/7109/es/

[5] A. Suárez, "Análisis de métodos de procesamiento de imágenes estereoscópicas forestales," Proyecto fin de máster en ingeniería informática para la industria, Universidad Complutense de Madrid, España, 2009.

[6] A. de la Escalera Hueso, Visión por computador: fundamentos y métodos. Pearson Educación, 2001. 